\title{
Estimation of the Bivariate Kumaraswamy Lifetime Distribution under Progressive Type-I Censoring
}

\author{
Hanan. M. Aly ${ }^{1}$, Hiba. Z. Muhammed², and Ola. A. Abuelamayem*1 \\ ${ }^{1}$ Department of Statistics, Faculty of Economics and Political Science, Cairo University, Cairo, Egypt \\ ${ }^{2}$ Department of Mathematical Statistics, Faculty of Graduate Studies of Statistical Research, Cairo \\ University, Cairo, Egypt
}

\begin{abstract}
Analyzing time to event data arises in a number of fields such as Biology and Engineering. A common feature of this data is that, the exact failure time for all units may not be observable. Accordingly, several types of censoring were presented. Progressive censoring allows units to be randomly removed before the terminal point of the experiment. Marshall-Olkin bivariate lifetime distribution was first introduced in 1967 using the exponential distribution. Recently, bivariate Marshall-Olkin Kumaraswamy lifetime distribution was derived. This paper derives the likelihood function under progressive type-I censoring for the bivariate Marshall-Olkin family in general and applies it on the bivariate Kumaraswamy lifetime distribution. Maximum likelihood estimators of model parameters were derived. Simulation study and a real data set are presented to illustrate the proposed procedure. Absolute bias, mean square error, asymptotic confidence intervals, confidence width and coverage probability are obtained. Simulation results indicate that the mean square error is smaller and confidence width is narrower and more precise when number of removals gets smaller. Also, increasing the terminal point of the experiment results in reducing the mean square error and confidence width.
\end{abstract}

Keywords bivariate Marshall-Olkin; maximum likelihood estimation.

\section{Introduction}

In many real life situations, there is a great need to understand and study the distribution of two variables interacting together and not each one separately. There are several examples in Biology such as; the study of twins, study of blindness in the left and right eye, failure time of the left and right kidney, the age at death of parent and child in a genetic study, studying the relation between heights and weights for a children, and studying the relation between blood pressure and body weight for a patient. Also, in reliability engineering to model the lifetime of a twine-engine plane, warranty polices based on failure time and warranty servicing time, as well as, different lifetime models, for example, shock model, maintenance model, bivariate longevity model.

Different bivariate family distributions were constructed. See for example, Marshall and Olkin (1967), Sankaran and Gleeja (2006), Regoli (2009), Mirhosseini et al. (2015). Great review for the bivariate distributions can be found in Balakrishnan and Lai (2009). Marshall and Olkin (1967) presented a bivariate exponential distribution with exponential marginals and loss of memory property. This bivariate distribution was used to represent the shock model. Several distributions were constructed by the same way, for example, Muhammed (2016) introduced the

\footnotetext{
${ }^{*}$ Corresponding author. E-mail: ola.abuelamayem@feps.edu.eg.
} 
bivariate inverse Weibull distribution. Different extensions for the Marshall-Olkin family were presented, see for example, Sarhan and Balakrishnan (2007), Jose et al. (2011), Li and Pellerey (2011), Ozkut and Bayramoglu (2014), and Davarzani et al. (2015). Barreto-Souza and Lemonte (2013) introduced the bivariate Kumaraswamy (BK) distribution, which can be applied in several reliability models like shock model, maintenance model and stress model. Özel (2015) showed that the BK model fits the earthquakes well.

There are several types of censoring, type-I (see Angali et al. (2014)), censored data with concomitant of order statistics (see Chen et al. (2000), Hanagal and Sharma (2015)). and hybrid random censoring (see Hanagal (1997)). The previous mentioned types of censoring do not allow any unit to be randomly removed at a point different from the terminal point of the experiment. Progressive censoring deals with this disadvantage by allowing units to be randomly removed during the experiment. Progressive censoring was first introduced in the univariate case by Cohen (1963). For more details, see Balakrishnan and Aggarwala (2000) and Lin and Balakrishnan (2011). To the best of our knowledge, the likelihood function in the bivariate case under progressive censoring was not derived yet. The advantage of this type of censoring is reducing cost and time of the experiment by allowing units to be randomly removed during the experiment. Because of the great importance of bivariate distributions in lifetime studies and the wide applicability of bivariate Marshall-Olkin family illustrated above. This paper, considers bivariate Marshall-Olkin family and derives the likelihood under progressive type-I censoring in general. The derived likelihood function is applied on the bivariate Kumaraswamy distribution.

The paper is organized as follows. The model is described in Section 2. The derivation of the likelihood function is done in Section 3. The maximum likelihood estimators of the model parameters and Fisher information matrix are presented in Section 4. In Section 5, numerical analysis including both simulated data and a real data set is done. Finally, the paper is concluded in Section 6.

\section{The Model under Type-I Censoring}

Marshall and Olkin (1967) was the first to introduce a bivariate exponential distribution with exponential marginals, loss of memory property and with applicability in real life. Using the same procedure introduced by Marshall and Olkin (1967) several distributions were derived. Recently, Barreto-Souza and Lemonte (2013) derived the BK distribution as follows.

Let $Z_{1}, Z_{2}$ and $Z_{3}$ be independent random variables such that $Z_{i}$ follows Kumraswamy $\left(\alpha_{i}, \beta\right)$, with probability density function given by:

$$
f_{k}(z ; \alpha, \beta)=\alpha \beta z^{\beta-1}\left(1-z^{\beta}\right)^{\alpha},
$$

where $\alpha_{i}>0, i=1,2,3$, and $\beta>0$ are shape parameters. Assume that $X_{1}=\min \left(Z_{1}, Z_{3}\right)$ and $X_{2}=\min \left(Z_{2}, Z_{3}\right)$, then the bivariate vector $\left(X_{1}, X_{2}\right)$ follows BK with the joint probability density function as follows:

$$
f_{X_{1}, X_{2}}\left(x_{1}, x_{2}\right)= \begin{cases}f_{1}\left(x_{1}, x_{2}\right) & 0<x_{1}<x_{2}<1 \\ f_{2}\left(x_{1}, x_{2}\right) & 0<x_{2}<x_{1}<1 \\ f_{3}(x), & 0<x_{1}=x_{2}=x<1\end{cases}
$$


where

$$
\begin{aligned}
f_{1}\left(x_{1}, x_{2}\right) & =\alpha_{1}\left(\alpha_{2}+\alpha_{3}\right) \beta^{2} x_{1}^{\beta-1}\left(1-x_{1}^{\beta}\right)^{\alpha_{1}-1} x_{2}^{\beta-1}\left(1-x_{2}^{\beta}\right)^{\left(\alpha_{2}+\alpha_{3}-1\right)}, \\
f_{2}\left(x_{1}, x_{2}\right) & =\alpha_{2}\left(\alpha_{1}+\alpha_{3}\right) \beta^{2} x_{1}^{\beta-1}\left(1-x_{1}^{\beta}\right)^{\alpha_{1}+\alpha_{3}-1} x_{2}^{\beta-1}\left(1-x_{2}^{\beta}\right)^{\left(\alpha_{2}-1\right)}, \\
f_{3}(x) & =\alpha_{3} \beta x^{\beta-1}\left(1-x^{\beta}\right)^{\alpha_{1}+\alpha_{2}+\alpha_{3}-1} .
\end{aligned}
$$

Hanagal (1992) presented type-I censoring in the bivariate case. Let $\left\{\left(x_{11}, x_{21}\right), \ldots,\left(x_{1 N}, x_{2 N}\right)\right\}$ be a random sample of size $N$, and $t$ be a pre-specified time at which the experiment is terminated. The lifetimes associated with the $i^{\text {th }}$ pair are given by:

$$
\left(x_{1 i}, x_{2 i}\right)= \begin{cases}\left(x_{1 i}, x_{2 i}\right) & \max \left(x_{1 i}, x_{2 i}\right)<t \\ \left(x_{1 i}, t\right) & x_{1 i}<t<x_{2 i} \\ \left(t, x_{2 i}\right) & x_{2 i}<t<x_{1 i} \\ (t, t) & \min \left(x_{1 i}, x_{2 i}\right)>t .\end{cases}
$$

The likelihood function has the following form:

$$
L=\prod_{i=1}^{n_{1}} f_{1}\left(x_{1 i}, x_{2 i}\right) \prod_{i=1}^{n_{2}} f_{2}\left(x_{1 i}, x_{2 i}\right) \prod_{i=1}^{n_{3}} f_{3}\left(x_{i}\right) \prod_{i=1}^{n_{4}} f_{4}\left(x_{1 i}, t\right) \prod_{i=1}^{n_{5}} f_{5}\left(t, x_{2 i}\right)\{[S(t, t)]\}^{n_{6}}
$$

where $f_{1}\left(x_{1 i}, x_{2 i}\right)$ corresponds to the case where $0<x_{1 i}<x_{2 i} \leq t ; f_{2}\left(x_{1 i}, x_{2 i}\right)$ corresponds to the case where $0<x_{1 i}<x_{2 i} \leq t ; f_{3}\left(x_{i}\right)$ corresponds to the case where $0<x_{1 i}=x_{2 i}=x_{i} \leq t$; $f_{4}\left(x_{1 i}, t\right)=\lim _{\delta x_{1 i} \rightarrow 0} \frac{P\left(x_{1 i}<X_{1 i}<x_{1 i}+\delta x_{1 i} \mid X_{2 i}>t\right) P\left(X_{2 i}>t\right)}{\delta x_{1 i}}=\alpha_{1} \beta x_{1 i}^{\beta-1}\left(1-x_{1 i}^{\beta}\right)^{\alpha_{1}-1}\left(1-t^{\beta}\right)^{\alpha_{2}+\alpha_{3}}$, $f_{5}\left(t, x_{2 i}\right)=\lim _{\delta x_{2 i} \rightarrow 0} \frac{P\left(x_{2 i}<X_{2 i}<x_{2 i}+\delta x_{2 i} \mid X_{1 i}>t\right) P\left(X_{1 i}>t\right)}{\delta x_{2 i}}=\alpha_{2} \beta x_{2 i}^{\beta-1}\left(1-x_{2 i}^{\beta}\right)^{\alpha_{2}-1}\left(1-t^{\beta}\right)^{\alpha_{1}+\alpha_{3}} ;$ $S(t, t)=P\left(X_{1 i}>t, X_{2 i}>t\right) ; n_{i}$ are the number of observations falling in the range corresponding to $f_{i}, i=1,2, \ldots, 5$, respectively and $n_{6}$ is the number corresponding to $S$.

\section{Derivation of the Likelihood Function under Progressive Cen- soring}

In this section we derive the likelihood function for the Marshall-Olkin family under progressive type-I censoring in general.

Suppose $\left\{\left(x_{11}, x_{21}\right), \ldots,\left(x_{1 N}, x_{2 N}\right)\right\}$ is a random sample of size $N$, and $t_{j}$ be a pre-specified times, such that $\left(x_{1}, x_{2}\right)$ is independent from $t_{j}, j=1, \ldots, m$. The censoring here occurs progressively in $m$ stages at pre-specified times $t_{j}$, such that $t_{j}>t_{j-1}$.

In the first stage, $N$ units are observed until the first censoring time $t_{1}$ when the failed $n_{1}$ units are counted. From the survived units (i.e. $x_{1}>t_{1}$ and $x_{2}>t_{1}$ ) $R_{1}$ pairs of units are randomly removed from the experiment. The remaining $N-n_{1}-R_{1}$ units entered the second stage. Similarly, in the second stage $N-n_{1}-R_{1}$ are observed till the second censoring time $t_{2}$ when the failed $n_{2}$ units are counted. From the survived units (i.e. $x_{1}>t_{2}$ and $\left.x_{2}>t_{2}\right) R_{2}$ pairs of units are randomly removed from the experiment and so on. At time $t_{m}$, the failed $n_{m}$ units are counted. All the remaining survived pairs of units are removed (i.e $\left.R_{m}=N-\sum_{j=1}^{m} n_{j}-\sum_{j=1}^{m-1} R_{j}\right)$ and the experiment terminates.

The failed units in each stage are divided according to 5 different cases illustrated as follows: Case 1: $x_{1 i j}<x_{2 i j} \leq t_{j}$, case 2: $x_{2 i j}<x_{1 i j} \leq t_{j}$, case $3: x_{1 i j}=x_{2 i j}=x_{i j} \leq t_{j}$, case 4 : 
$x_{1 i j} \leq t_{j}<x_{2 i j}$ and case 5: $x_{2 i j} \leq t_{j}<x_{1 i j}$. To reflect this division we define 5 indicator functions as follows:

$$
\delta_{k i j}= \begin{cases}1, & \text { if } i j \text { observation belongs to case } k \\ 0, & \text { otherwise }\end{cases}
$$

The likelihood function is derived to be as follows:

$$
\begin{gathered}
L_{1}=\prod_{j=1}^{m} \prod_{i=1}^{n_{j}}\left\{\left[f_{1}\left(x_{1 i j}, x_{2 i j}\right)\right]^{\delta_{1 i j}}\left[f_{2}\left(x_{1 i j}, x_{2 i j}\right)\right]^{\delta_{2 i j}}\left[f_{3}\left(x_{i j}\right)\right]^{\delta_{3 i j}}\right. \\
\left.\left[f_{4}\left(x_{1 i j}, t_{j}\right)\right]^{\delta_{4 i j}}\left[f_{5}\left(t_{j}, x_{2 i j}\right)\right]^{\delta_{5 i j}}\right\}\left[S\left(t_{j}, t_{j}\right)\right]^{R_{j}}
\end{gathered}
$$

Applying on the bivariate Kumaraswamy lifetime distribution, the likelihood function takes the following form:

$$
\begin{aligned}
& L_{1}=\prod_{j=1}^{m} \prod_{i=1}^{n_{j}}\left\{\alpha_{1}^{\delta_{1 i j}+\delta_{4 i j}} \alpha_{2}^{\delta_{2 i j}+\delta_{5 i j}} \alpha_{3}^{\delta_{3 i j}} \beta^{2 \delta_{1 i j}+2 \delta_{2 i j}+\delta_{3 i j}+\delta_{4 i j}+\delta_{5 i j}}\left(\alpha_{2}+\alpha_{3}\right)^{\delta_{1 i j}}\left(\alpha_{1}+\alpha_{3}\right)^{\delta_{2 i j}}\right. \\
& x_{1 i j}^{(\beta-1)\left(\delta_{1 i j}+\delta_{2 i j}+\delta_{4 i j}\right)} x_{2 i j}^{(\beta-1)\left(\delta_{1 i j}+\delta_{2 i j}+\delta_{5 i j}\right)} x_{i j}^{(\beta-1) \delta_{3 i j}} \\
&\left(1-x_{1 i j}^{\beta}\right)^{\delta_{1 i j}\left(\alpha_{1}-1\right)+\delta_{2 i j}\left(\alpha_{1}+\alpha_{3}-1\right)+\left(\alpha_{1}-1\right) \delta_{4 i j}} \\
&\left(1-x_{2 i j}^{\beta}\right)^{\delta_{2 i j}\left(\alpha_{2}-1\right)+\delta_{1 i j}\left(\alpha_{2}+\alpha_{3}-1\right)+\left(\alpha_{2}-1\right) \delta_{5 i j}}\left(1-x_{i j}^{\beta}\right)^{\delta_{3 i j}\left(\alpha_{1}+\alpha_{2}+\alpha_{3}-1\right)} \\
&\left.\left(1-t_{j}^{\beta}\right)^{\left(\alpha_{2}+\alpha_{3}\right) \delta_{4 i j}+\left(\alpha_{1}+\alpha_{3}\right) \delta_{5 i j}}\right\}\left(1-t_{j}^{\beta}\right)^{\left(\alpha_{1}+\alpha_{2}+\alpha_{3}\right) R_{j}} .
\end{aligned}
$$

\section{Maximum Likelihood Estimation}

In this section, the maximum likelihood estimators (MLEs) of the unknown parameters are obtained by maximizing the logarithm of the likelihood function derived in Section 3. Moreover, the observed Fisher information matrix is obtained. The confidence intervals are constructed using the asymptotic properties of the MLEs.

The derivatives of the log-likelihood with respect to the unknown parameters are as follows:

$$
\begin{aligned}
\frac{\partial \log L_{1}}{\partial \alpha_{1}}= & \frac{1}{\alpha_{1}} \sum_{j=1}^{m} \sum_{i=1}^{n_{j}}\left(\delta_{1 i j}+\delta_{4 i j}\right)+\frac{1}{\alpha_{1}+\alpha_{3}} \sum_{j=1}^{m} \sum_{i=1}^{n_{j}} \delta_{2 i j}+\sum_{j=1}^{m} \sum_{i=1}^{n_{j}}\left(\delta_{1 i j}+\delta_{2 i j}+\delta_{4 i j}\right) \log \left(1-x_{1 i j}^{\beta}\right) \\
& +\sum_{j=1}^{m} \sum_{i=1}^{n_{j}} \delta_{3 i j} \log \left(1-x_{i j}^{\beta}\right)+\sum_{j=1}^{m} \sum_{i=1}^{n_{j}} \delta_{5 i j} \log \left(1-t_{j}^{\beta}\right)+\sum_{j=1}^{m} R_{j} \log \left(1-t_{j}^{\beta}\right), \\
\frac{\partial \log L_{1}}{\partial \alpha_{2}}= & \frac{1}{\alpha_{2}} \sum_{j=1}^{m} \sum_{i=1}^{n_{j}}\left(\delta_{2 i j}+\delta_{5 i j}\right)+\frac{1}{\alpha_{2}+\alpha_{3}} \sum_{j=1}^{m} \sum_{i=1}^{n_{j}} \delta_{1 i j}+\sum_{j=1}^{m} \sum_{i=1}^{n_{j}}\left(\delta_{1 i j}+\delta_{2 i j}+\delta_{5 i j}\right) \log \left(1-x_{2 i j}^{\beta}\right) \\
& +\sum_{j=1}^{m} \sum_{i=1}^{n_{j}} \delta_{3 i j} \log \left(1-x_{i j}^{\beta}\right)+\sum_{j=1}^{m} \sum_{i=1}^{n_{j}} \delta_{4 i j} \log \left(1-t_{j}^{\beta}\right)+\sum_{j=1}^{m} R_{j} \log \left(1-t_{j}^{\beta}\right), \\
\frac{\partial \log L_{1}}{\partial \alpha_{3}}= & \frac{1}{\alpha_{2}+\alpha_{3}} \sum_{j=1}^{m} \sum_{i=1}^{n_{j}} \delta_{1 i j}+\frac{1}{\alpha_{1}+\alpha_{3}} \sum_{j=1}^{m} \sum_{i=1}^{n_{j}} \delta_{2 i j}+\frac{1}{\alpha_{3}} \sum_{j=1}^{m} \sum_{i=1}^{n_{j}} \delta_{3 i j}+\sum_{j=1}^{m} \sum_{i=1}^{n_{j}} \delta_{2 i j} \log \left(1-x_{1 i j}^{\beta}\right)
\end{aligned}
$$




$$
\begin{aligned}
& +\sum_{j=1}^{m} \sum_{i=1}^{n_{j}} \delta_{1 i j} \log \left(1-x_{2 i j}^{\beta}\right)+\sum_{j=1}^{m} \sum_{i=1}^{n_{j}} \delta_{3 i j} \log \left(1-x_{i j}^{\beta}\right) \sum_{j=1}^{m} \sum_{i=1}^{n_{j}}\left(\delta_{4 i j}+\delta_{5 i j}\right) \log \left(1-t_{j}^{\beta}\right) \\
& +\sum_{j=1}^{m} R_{j} \log \left(1-t_{j}^{\beta}\right), \\
\frac{\partial \log L_{1}}{\partial \beta}= & A_{1}+B_{1}+A_{2}+B_{2}+A_{3}+B_{3}+A_{4}+C_{1}+C_{2},
\end{aligned}
$$

where

$$
\begin{aligned}
& A_{1}=\frac{1}{\beta} \sum_{j=1}^{m} \sum_{i=1}^{n_{j}}\left(2 \delta_{1 i j}+2 \delta_{2 i j}+\delta_{3 i j}+\delta_{4 i j}+\delta_{5 i j}\right) \\
& A_{2}=-\sum_{j=1}^{m} \sum_{i=1}^{n_{j}} \frac{\left\{\left(\alpha_{1}-1\right) \delta_{1 i j}+\left(\alpha_{1}+\alpha_{3}-1\right) \delta_{2 i j}+\left(\alpha_{1}-1\right) \delta_{4 i j}\right\} x_{1 i j}^{\beta} \log x_{1 i j}}{1-x_{1 i j}^{\beta}} \\
& A_{3}=-\sum_{j=1}^{m} \sum_{i=1}^{n_{j}} \frac{\left\{\left(\alpha_{2}+\alpha_{3}-1\right) \delta_{1 i j}+\left(\alpha_{2}-1\right) \delta_{2 i j}+\left(\alpha_{2}-1\right) \delta_{5 i j}\right\} x_{2 i j}^{\beta} \log x_{2 i j}}{1-x_{2 i j}^{\beta}} \\
& A_{4}=-\left(\alpha_{1}+\alpha_{2}+\alpha_{3}-1\right) \sum_{j=1}^{m} \sum_{i=1}^{n_{j}} \frac{\delta_{3 i j} x_{i j}^{\beta} \log x_{i j}}{1-x_{i j}^{\beta}} \\
& B_{1}=\sum_{j=1}^{m} \sum_{i=1}^{n_{j}}\left\{\delta_{1 i j}+\delta_{2 i j}+\delta_{4 i j}\right\} \log x_{1 i j}, \\
& B_{2}=\sum_{j=1}^{m} \sum_{i=1}^{n_{j}}\left(\delta_{1 i j}+\delta_{2 i j}+\delta_{5 i j}\right) \log x_{2 i j}, \\
& B_{3}=\sum_{j=1}^{m} \sum_{i=1}^{n_{j}} \delta_{3 i j} \log x_{i j}, \\
& C_{1}=-\sum_{j=1}^{m} \sum_{i=1}^{n_{j}} \frac{\left\{\left(\alpha_{2}+\alpha_{3}\right) \delta_{4 i j}+\left(\alpha_{1}+\alpha_{3}\right) \delta_{5 i j}\right\} t_{j}^{\beta} \log t_{j}}{1-t_{j}^{\beta}}, \text { and } \\
& C_{2}=-\left(\alpha_{1}+\alpha_{2}+\alpha_{3}\right) \sum_{j=1}^{m} \frac{R_{j} t_{j}^{\beta} \log t_{j}}{1-t_{j}^{\beta}} .
\end{aligned}
$$

If $\sum_{j=1}^{m} \sum_{i=1}^{n_{j}} \delta_{k i j}>0, k=1,2,3$, the equations can be solved numerically. $\sum_{j=1}^{m} \sum_{i=1}^{n_{j}} \delta_{k i j}$, $k=4,5$ and $n_{6}$ can be equal to 0 , as this will lead to the complete case. Bemis et al. (1972) explained the conditions for the bivariate exponential distribution in the complete case, and they reached the same conditions to have separate estimates for all the parameters.

Let the Fisher information matrix be denoted by

$$
\mathrm{I}\left(\alpha_{1}, \alpha_{2}, \alpha_{3}, \beta\right)=\left[\begin{array}{cccc}
I_{11} & 0 & I_{13} & I_{14} \\
0 & I_{22} & I_{23} & I_{24} \\
I_{13} & I_{23} & I_{33} & I_{34} \\
I_{14} & I_{24} & I_{34} & I_{44}
\end{array}\right]
$$


We have

$$
\begin{aligned}
& I_{11}=-\frac{\partial^{2} \log L_{1}}{\partial \alpha_{1}^{2}}=\frac{1}{\alpha_{1}^{2}} \sum_{j=1}^{m} \sum_{i=1}^{n_{j}}\left(\delta_{1 i j}+\delta_{4 i j}\right)+\frac{1}{\left(\alpha_{1}+\alpha_{3}\right)^{2}} \sum_{j=1}^{m} \sum_{i=1}^{n_{j}} \delta_{2 i j}, \\
& I_{13}=-\frac{\partial^{2} \log L_{1}}{\partial \alpha_{1} \partial \alpha_{3}}=\frac{1}{\left(\alpha_{1}+\alpha_{3}\right)^{2}} \sum_{j=1}^{m} \sum_{i=1}^{n_{j}} \delta_{2 i j} \\
& I_{14}=-\frac{\partial^{2} \log L_{1}}{\partial \alpha_{1} \partial \beta}=\sum_{j=1}^{m} \sum_{i=1}^{n_{j}} \frac{\left(\delta_{1 i j}+\delta_{2 i j}+\delta_{4 i j}\right) x_{1 i j}^{\beta} \log x_{1 i j}}{1-x_{1 i j}^{\beta}}+\sum_{j=1}^{m} \sum_{i=1}^{n_{j}} \frac{\delta_{3 i j} x_{i j}^{\beta} \log x_{i j}}{1-x_{i j}^{\beta}} \\
& +\sum_{j=1}^{m} \sum_{i=1}^{n_{j}} \frac{\delta_{5 i j} t_{j}^{\beta} \log t_{j}}{1-t_{j}^{\beta}}+\sum_{j=1}^{m} \frac{R_{j} t_{j}^{\beta} \log t_{j}}{1-t_{j}^{\beta}} \\
& I_{22}=-\frac{\partial^{2} \log L_{1}}{\partial \alpha_{2}^{2}}=\frac{1}{\left(\alpha_{2}+\alpha_{3}\right)^{2}} \sum_{j=1}^{m} \sum_{i=1}^{n_{j}} \delta_{1 i j}+\frac{1}{\alpha_{2}^{2}} \sum_{j=1}^{m} \sum_{i=1}^{n_{j}}\left(\delta_{2 i j}+\delta_{5 i j}\right) \text {, } \\
& I_{23}=-\frac{\partial^{2} \log L_{1}}{\partial \alpha_{2} \partial \alpha_{3}}=\frac{1}{\left(\alpha_{2}+\alpha_{3}\right)^{2}} \sum_{j=1}^{m} \sum_{i=1}^{n_{j}} \delta_{1 i j}, \\
& I_{24}=-\frac{\partial^{2} \log L_{1}}{\partial \alpha_{2} \partial \beta}=\sum_{j=1}^{m} \sum_{i=1}^{n_{j}} \frac{x_{i j}^{\beta} \log x_{i j} \delta_{3 i j}}{1-x_{i j}^{\beta}}+\sum_{j=1}^{m} \sum_{i=1}^{n_{j}} \frac{\left(\delta_{1 i j}+\delta_{2 i j}+\delta_{5 i j}\right) x_{2 i j}^{\beta} \log x_{2 i j}}{1-x_{2 i j}^{\beta}} \\
& +\sum_{j=1}^{m} \sum_{i=1}^{n_{j}} \frac{\delta_{4 i j} t_{j}^{\beta} \log t_{j}}{1-t_{j}^{\beta}}+\sum_{j=1}^{m} \frac{R_{j} t_{j}^{\beta} \log t_{j}}{1-t_{j}^{\beta}} \\
& I_{33}=-\frac{\partial^{2} \log L_{1}}{\partial \alpha_{3}^{2}}=\frac{1}{\left(\alpha_{2}+\alpha_{3}\right)^{2}} \sum_{j=1}^{m} \sum_{i=1}^{n_{j}} \delta_{1 i j}+\frac{1}{\left(\alpha_{1}+\alpha_{3}\right)^{2}} \sum_{j=1}^{m} \sum_{i=1}^{n_{j}} \delta_{2 i j}+\frac{1}{\alpha_{3}^{2}} \sum_{j=1}^{m} \sum_{i=1}^{n_{j}} \delta_{3 i j} \text {, } \\
& I_{34}=-\frac{\partial^{2} \log L_{1}}{\partial \alpha_{3} \partial \beta}=\sum_{j=1}^{m} \sum_{i=1}^{n_{j}} \frac{\delta_{2 i j} x_{1 i j}^{\beta} \log x_{1 i j}}{1-x_{1 i j}^{\beta}}+\sum_{j=1}^{m} \sum_{i=1}^{n_{j}} \frac{\delta_{1 i j} x_{2 i j}^{\beta} \log x_{2 i j}}{1-x_{2 i j}^{\beta}}+\sum_{j=1}^{m} \sum_{i=1}^{n_{j}} \frac{\delta_{3 i j} x_{i j}^{\beta} \log x_{i j}}{1-x_{i j}^{\beta}} \\
& +\sum_{j=1}^{m} \sum_{i=1}^{n_{j}} \frac{\left(\delta_{4 i j}+\delta_{5 i}\right) t_{j}^{\beta} \log t_{j}}{1-t_{j}^{\beta}}+\sum_{j=1}^{m} \frac{R_{j} t_{j}^{\beta} \log t_{j}}{1-t_{j}^{\beta}} \text {, and } \\
& I_{44}=-\frac{\partial^{2} \log L_{1}}{\partial \beta^{2}}=D_{1}+D_{2}+D_{3}+D_{4}+E_{1}+E_{2} \text {, }
\end{aligned}
$$

where

$$
\begin{aligned}
& D_{1}=\frac{1}{\beta^{2}} \sum_{j=1}^{m} \sum_{i=1}^{n_{j}}\left(2 \delta_{1 i j}+2 \delta_{2 i j}+\delta_{3 i j}+\delta_{4 i j}+\delta_{5 i j}\right) \\
& D_{2}=\sum_{j=1}^{m} \sum_{i=1}^{n_{j}} \frac{\left\{\left(\alpha_{1}-1\right) \delta_{1 i j}+\left(\alpha_{1}+\alpha_{3}-1\right) \delta_{2 i j}+\left(\alpha_{1}-1\right) \delta_{4 i j}\right\} x_{1 i j}^{\beta}\left(\log x_{1 i j}\right)^{2}}{\left(1-x_{1 i j}^{\beta}\right)^{2}}, \\
& D_{3}=\sum_{j=1}^{m} \sum_{i=1}^{n_{j}} \frac{\left\{\left(\alpha_{2}+\alpha_{3}-1\right) \delta_{1 i j}+\left(\alpha_{2}-1\right) \delta_{2 i j}+\left(\alpha_{2}-1\right) \delta_{5 i j}\right\} x_{2 i j}^{\beta}\left(\log x_{2 i j}\right)^{2}}{\left(1-x_{2 i j}^{\beta}\right)^{2}},
\end{aligned}
$$




$$
\begin{aligned}
& D_{4}=\left(\alpha_{1}+\alpha_{2}+\alpha_{3}-1\right) \sum_{j=1}^{m} \sum_{i=1}^{n_{j}} \frac{\delta_{3 i j}\left(\log x_{i j}\right)^{2} x_{i j}^{\beta}}{\left(1-x_{i j}^{\beta}\right)^{2}}, \\
& E_{1}=\sum_{j=1}^{m} \sum_{i=1}^{n_{j}} \frac{\left\{\left(\alpha_{2}+\alpha_{3}\right) \delta_{4 i j}+\left(\alpha_{1}+\alpha_{3}\right) \delta_{5 i j}\right\}\left(\log t_{j}\right)^{2} t_{j}^{\beta}}{\left(1-t_{j}^{\beta}\right)^{2}}, \text { and } \\
& E_{2}=\left(\alpha_{1}+\alpha_{2}+\alpha_{3}\right) \sum_{j=1}^{m} \frac{R_{j}\left(\log t_{j}\right)^{2} t_{j}^{\beta}}{\left(1-t_{j}^{\beta}\right)^{2}} .
\end{aligned}
$$

Using the asymptotic distribution of the MLEs, the confidence intervals can be obtained as follows:

$$
\hat{\lambda} \pm z_{\frac{\gamma}{2}} \sqrt{\operatorname{var}(\hat{\lambda})}
$$

where $\hat{\lambda}=\left(\hat{\alpha_{1}}, \hat{\alpha_{2}}, \hat{\alpha_{3}}, \hat{\beta}\right), \operatorname{va} r(\hat{\lambda})$ is the estimated variance, and $z_{\frac{\gamma}{2}}$ is the upper ${ }^{\text {th }}$ percentile of the standard normal table.

\section{$5 \quad$ Numerical Results}

In this section, a simulation study is carried out to investigate the performance of the derived likelihood function under different schemes using different arbitrary chosen values of $t, R$ and using different $N$, and also, using different populations. Moreover, a real data set is analyzed.

\subsection{Simulation Results}

Here, the following schemes are considered:

- Scheme 1: $N=40, m=2, R_{1}=3$;

- Scheme 2: $N=40, m=2, R_{1}=5$;

- Scheme 3: $N=70, m=3, R_{1}=R_{2}=5$;

- Scheme 4: $N=70, m=3, R_{1}=R_{2}=7$.

$\mathrm{R}$ was used with 5000 replications, the results are presented in Table 1 and Table 2 for scheme 1 to scheme 4. Absolute Bias (ABias), Mean Square Error (MSE), Confidence Width $(\mathrm{CW})$ and Coverage Probability $(\mathrm{CP})$ are obtained for each scheme. The results are analyzed by two different ways. First, consider the following division to study the effect of increasing the number of removals

- Set 1: schemes 1 and 3;

- Set 2: schemes 2 and 4 .

It can be seen that for the majority of the cases MSEs are smaller in set 1 than that in set 2 . For example, comparing scheme 1 and scheme $2, t_{1}=0.05$ and $t_{2}=0.4$ and all parameters equal to 1. MSE for $\alpha_{1}$ decreased from 0.265 in scheme 2 to 0.242 in scheme 1 . Also, CWs are narrower in set 1 than that in set 2. For the same example above CW decreased from 1.968 to 1.884 . Moreover, ABias decreases for more than half of the cases in set 1 than that in set 2. For the same example MSE decreased from 0.116 to 0.106 .

Second, studying the effect of increasing the value of the terminal point of the experiment as follows:

- Set 1: $t_{1}=0.05$ and $t_{2}=0.4$ (i.e. Table 1 );

- Set 2: $t_{1}=0.1$ and $t_{2}=0.6$ (i.e. Table 1 );

- Set 3: $t_{1}=0.09, t_{2}=0.15$ and $t_{3}=0.4$ (i.e. Table 2 ); 
Table 1: The results for schemes 1 and 2 .

\begin{tabular}{|c|c|c|c|c|c|c|c|c|c|c|}
\hline & & & \multicolumn{4}{|c|}{$\left(t_{1}, t_{2}\right)=(0.05,0.4)$} & \multicolumn{4}{|c|}{$\left(t_{1}, t_{2}\right)=(0.1,0.6)$} \\
\hline & & & $\alpha_{1}$ & $\alpha_{2}$ & $\alpha_{3}$ & $\beta$ & $\alpha_{1}$ & $\alpha_{2}$ & $\alpha_{3}$ & $\beta$ \\
\hline \multirow{8}{*}{$\begin{array}{l}\alpha_{1}=0.5, \alpha_{2}=0.5 \\
\alpha_{3}=0.5, \beta=0.5\end{array}$} & \multirow{4}{*}{$R_{1}=3$} & ABias & 0.036 & 0.044 & 0.040 & 0.023 & 0.034 & 0.040 & 0.033 & 0.024 \\
\hline & & MSE & 0.046 & 0.048 & 0.044 & 0.012 & 0.036 & 0.038 & 0.033 & 0.011 \\
\hline & & $\mathrm{CW}$ & 0.832 & 0.840 & 0.804 & 0.412 & 0.734 & 0.744 & 0.702 & 0.392 \\
\hline & & $\mathrm{CP}$ & 0.934 & 0.938 & 0.940 & 0.949 & 0.943 & 0.942 & 0.948 & 0.949 \\
\hline & \multirow{4}{*}{$R_{1}=5$} & ABias & 0.047 & 0.042 & 0.036 & 0.023 & 0.038 & 0.038 & 0.037 & 0.023 \\
\hline & & MSE & 0.051 & 0.051 & 0.046 & 0.013 & 0.040 & 0.039 & 0.036 & 0.011 \\
\hline & & $\mathrm{CW}$ & 0.868 & 0.868 & 0.832 & 0.430 & 0.774 & 0.764 & 0.734 & 0.392 \\
\hline & & $\mathrm{CP}$ & 0.942 & 0.937 & 0.933 & 0.956 & 0.932 & 0.938 & 0.943 & 0.951 \\
\hline \multirow{8}{*}{$\begin{array}{l}\alpha_{1}=0.5, \alpha_{2}=0.4 \\
\alpha_{3}=0.4, \beta=0.5\end{array}$} & \multirow{4}{*}{$R_{1}=3$} & ABias & 0.039 & 0.034 & 0.032 & 0.027 & 0.035 & 0.027 & 0.023 & 0.023 \\
\hline & & MSE & 0.046 & 0.034 & 0.032 & 0.014 & 0.034 & 0.025 & 0.023 & 0.012 \\
\hline & & $\mathrm{CW}$ & 0.822 & 0.712 & 0.690 & 0.446 & 0.712 & 0.608 & 0.582 & 0.412 \\
\hline & & $\mathrm{CP}$ & 0.940 & 0.935 & 0.937 & 0.953 & 0.946 & 0.942 & 0.934 & 0.951 \\
\hline & \multirow{4}{*}{$R_{1}=5$} & ABias & 0.044 & 0.036 & 0.027 & 0.026 & 0.038 & 0.030 & 0.029 & 0.024 \\
\hline & & MSE & 0.050 & 0.036 & 0.033 & 0.014 & 0.037 & 0.027 & 0.025 & 0.012 \\
\hline & & $\mathrm{CW}$ & 0.858 & 0.734 & 0.702 & 0.446 & 0.744 & 0.632 & 0.608 & 0.412 \\
\hline & & $\mathrm{CP}$ & 0.938 & 0.931 & 0.927 & 0.948 & 0.945 & 0.939 & 0.942 & 0.951 \\
\hline \multirow{8}{*}{$\begin{array}{l}\alpha_{1}=1, \alpha_{2}=1 \\
\alpha_{3}=1, \beta=1\end{array}$} & \multirow{4}{*}{$R_{1}=3$} & ABias & 0.106 & 0.104 & 0.092 & 0.037 & 0.088 & 0.083 & 0.081 & 0.036 \\
\hline & & MSE & 0.242 & 0.241 & 0.213 & 0.036 & 0.161 & 0.158 & 0.138 & 0.028 \\
\hline & & CW & 1.884 & 1.880 & 1.774 & 0.734 & 1.534 & 1.524 & 1.418 & 0.644 \\
\hline & & $\mathrm{CP}$ & 0.936 & 0.938 & 0.934 & 0.950 & 0.944 & 0.943 & 0.947 & 0.946 \\
\hline & \multirow{4}{*}{$R_{1}=5$} & ABias & 0.116 & 0.111 & 0.113 & 0.046 & 0.085 & 0.094 & 0.082 & 0.034 \\
\hline & & MSE & 0.265 & 0.261 & 0.238 & 0.038 & 0.170 & 0.173 & 0.148 & 0.028 \\
\hline & & CW & 1.968 & 1.956 & 1.860 & 0.744 & 1.582 & 1.588 & 1.472 & 0.644 \\
\hline & & $\mathrm{CP}$ & 0.933 & 0.933 & 0.941 & 0.951 & 0.942 & 0.945 & 0.948 & 0.949 \\
\hline \multirow{8}{*}{$\begin{array}{l}\alpha_{1}=1.2, \alpha_{2}=1.2 \\
\alpha_{3}=1.2, \beta=1\end{array}$} & \multirow{4}{*}{$R_{1}=3$} & ABias & 0.109 & 0.119 & 0.108 & 0.032 & 0.101 & 0.105 & 0.099 & 0.033 \\
\hline & & MSE & 0.311 & 0.316 & 0.278 & 0.031 & 0.228 & 0.230 & 0.196 & 0.025 \\
\hline & & $\mathrm{CW}$ & 2.144 & 2.154 & 2.022 & 0.678 & 1.830 & 1.834 & 1.690 & 0.608 \\
\hline & & $\mathrm{CP}$ & 0.938 & 0.936 & 0.943 & 0.955 & 0.941 & 0.944 & 0.945 & 0.948 \\
\hline & \multirow{4}{*}{$R_{1}=5$} & ABias & 0.128 & 0.132 & 0.121 & 0.036 & 0.112 & 0.108 & 0.102 & 0.037 \\
\hline & & MSE & 0.342 & 0.346 & 0.304 & 0.032 & 0.250 & 0.247 & 0.211 & 0.026 \\
\hline & & $\mathrm{CW}$ & 2.238 & 2.248 & 2.108 & 0.690 & 1.908 & 1.900 & 1.758 & 0.620 \\
\hline & & $\mathrm{CP}$ & 0.939 & 0.936 & 0.941 & 0.948 & 0.945 & 0.943 & 0.942 & 0.948 \\
\hline
\end{tabular}

- Set 4: $t_{1}=0.05, t_{2}=0.25$ and $t_{3}=0.55$ (i.e. Table 2 ).

Increasing the terminal point of the experiment results in decreasing MSEs. For example, comparing set 1 and set 2, and setting all the parameters equal 1, MSE for $\alpha_{1}$ decreased from 0.242 to 0.161. Also, it leads to narrower and more precise CWs. For the same above example, CW decreases from 1.884 to 1.534 .

Finally, by summarizing all tables, it is clear that CIs includes the true values of the proposed parameters. Also, for the majority of the cases the coverage probability is above $93 \%$, and $\beta$ considers more satisfactory as its coverage probability is more close to $95 \%$. 
Table 2: The results for schemes 3 and 4 .

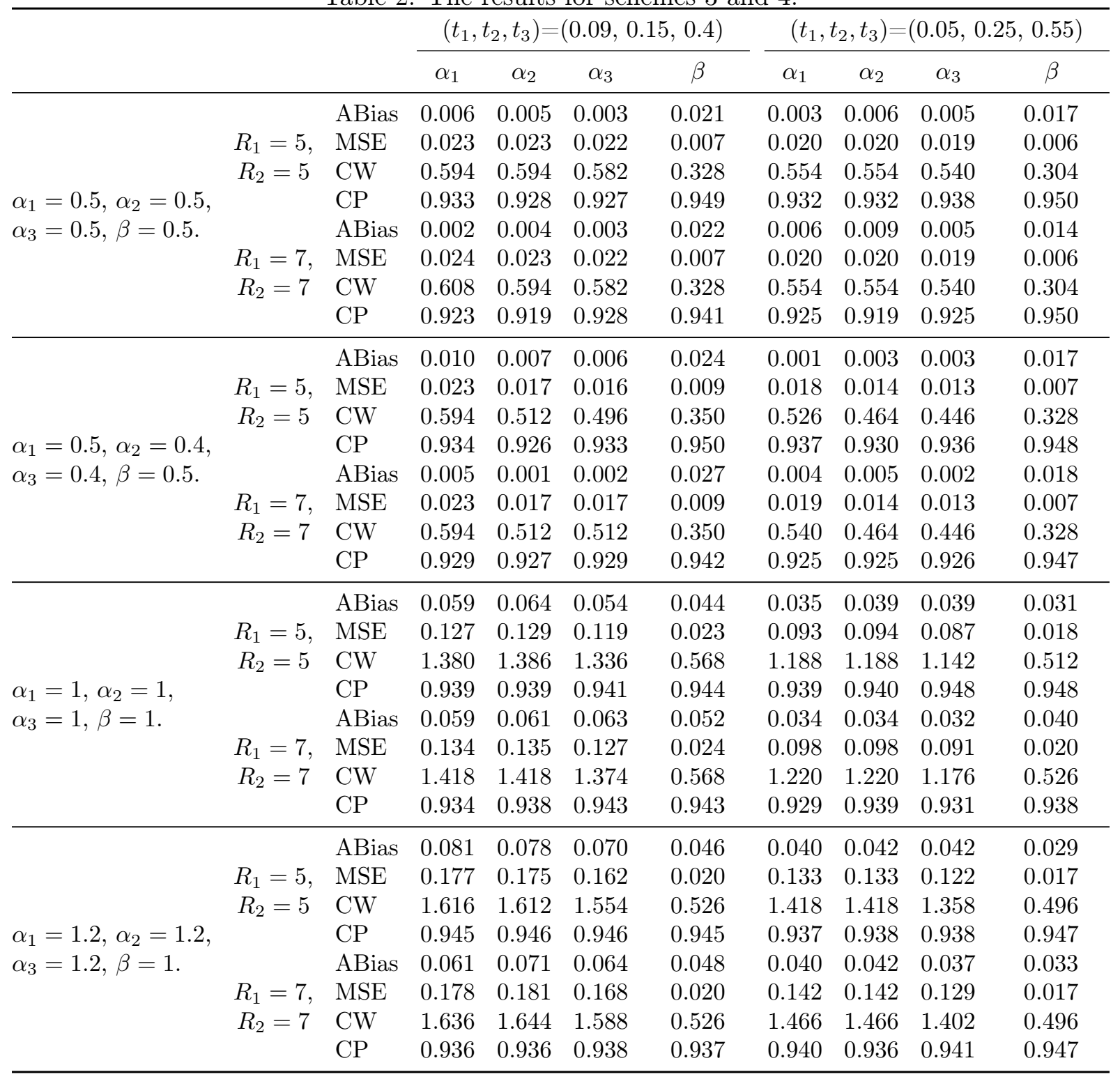

\subsection{A Real Life Data Set}

A real data set is obtained from Barreto-Souza and Lemonte (2013), it corresponds to football (soccer) data such that at least one goal scored by the home team and at least one goal scored directly from a penalty kick, a foul kick or any other direct kick by any team have been considered. Let $Z_{1}$ corresponds to the time in minutes of the first kick goal scored by any team, and $Z_{2}$ corresponds to the first goal of any type scored by the home team. All the data points have been divided by 90 . Accordingly, we analyze the proportion of time that the home team and any other team scored the first kick goal. Barreto-Souza and Lemonte (2013) computed the modified Cramer-von Mises and Anderson-Darling statistics. The values of the two statistics and their corresponding p-values showed that the BK model fits the data well. We will try to apply the data they used to illustrate our procedure and we consider the following two schemes: 
Table 3: Estimated parameters and CWs for the BK distribution.

\begin{tabular}{lccccc}
\hline & & $\hat{\alpha_{1}}$ & $\hat{\alpha_{2}}$ & $\hat{\alpha_{3}}$ & $\hat{\beta}$ \\
\hline$T_{1}=0.25$, & estimate & 0.396 & 1.394 & 1.067 & 1.284 \\
$T_{2}=0.55$ & CI & $(-0.038,0.830)$ & $(0.395,2.393)$ & $(0.273,1.861)$ & $(0.816,1.752)$ \\
& CW & 0.868 & 1.998 & 1.588 & 0.936 \\
$T_{1}=0.1$, & estimate & 0.585 & 1.394 & 1.088 & 1.293 \\
$T_{2}=0.4$ & CI & $(-0.088,1.258)$ & $(0.151,2.637)$ & $(0.096,2.080)$ & $(0.771,1.815)$ \\
& CW & 1.346 & 2.486 & 1.984 & 1.044 \\
\hline
\end{tabular}

- Scheme 1: $N=37, m=2, t_{1}=0.25$ and $t_{2}=0.55, R_{1}=3$ and $R_{2}=5$;

- Scheme 2: $N=37, m=2, t_{1}=0.1$ and $t_{2}=0.4, R_{1}=3$ and $R_{2}=11$.

CIs, CWs and MLEs are presented in Table 3 and it is observed that scheme 1 is more precise than scheme 2 . which is a logical result due to increasing the number of the analyzed failed units. Also, it supports the conclusion obtained from studying the effect of increasing the terminal point of the experiment in the simulation study. Also, for scheme 1, it can be seen that $Z_{1}$ follows Kumaraswamy $(1.463,1.284)$ and $Z_{2}$ follows Kumaraswamy $(2.462,1.284)$, and for scheme 2 , it can be seen that $Z_{1}$ follows Kumaraswamy $(1.673,1.293)$ and $Z_{2}$ follows Kumaraswamy $(2.482$, $1.293)$.

\section{Conclusion}

In this paper, the likelihood function was derived for the Marshall-Olkin family under progressive type-I censoring. The derived likelihood function was applied on the Marshall-Olkin bivariate Kumaraswamy lifetime distribution. Maximum likelihood estimation are obtained to estimate the unknown parameters. Moreover, asymptotic confidence intervals are constructed using observed Fisher information matrix. Simulation results indicated that for most of the cases MSEs are smaller, CWs are narrower and more precise when numbers of removals are less. Also, increasing the terminal point of the experiment results in reducing the MSEs and CWs. Moreover, a real data set is analyzed using different values of the terminal point which supports the last conclusion. The only limitation of this study is that the Kumaraswamy lifetime distribution is ranged between 0 and 1, which lead out time to be between 0 and 1 . But this is has no effect on the possibility to apply the results obtained on any lifetime distribution that ranged from 0 to infinity.

\section{References}

Angali KA, Latifi SM, Hanagal DD (2014). Bayesian estimation of bivariate exponential distributions based on Linex and quadratic loss functions: A survival approach with censored samples. Communications in Statistics-Simulation and Computation, 43(1): 31-44.

Balakrishnan N, Aggarwala R (2000). Progressive Censoring: Theory, Methods, and Applications. Springer Science \& Business Media.

Balakrishnan N, Lai CD (2009). Continuous Bivariate Distributions. Springer Science \& Business Media. 
Barreto-Souza W, Lemonte AJ (2013). Bivariate Kumaraswamy distribution: Properties and a new method to generate bivariate classes. Statistics, 47(6): 1321-1342.

Bemis BM, Bain LJ, Higgins JJ (1972). Estimation and hypothesis testing for the parameters of a bivariate exponential distribution. Journal of the American Statistical Association, 67(340): 927-929.

Chen D, Lu JC, Li CS, Park J (2000). Parameter estimation for bivariate shock models with singular distribution for censored data with concomitant order statistics. Australian $\&$ New Zealand Journal of Statistics, 42(3): 323-336.

Cohen AC (1963). Progressively censored samples in life testing. Technometrics, 5(3): 327-339.

Davarzani N, Parsian A, Peeters R (2015). Dependent right censorship in the MOMW distribution. Communications in Statistics-Theory and Methods, 44(11): 2222-2242.

Hanagal DD (1992). Some inference results in bivariate exponential distributions rased on censored samples. Communications in Statistics-Theory and Methods, 21(5): 1273-1295.

Hanagal DD (1997). Inference procedures in some bivariate exponential models under hybrid random censoring. Statistical Papers, 38(2): 167-189.

Hanagal DD, Sharma R (2015). Bayesian inference in Marshall-Olkin bivariate exponential shared Gamma frailty regression model under random censoring. Communications in Statistics-Theory and Methods, 44(1): 24-47.

Jose K, Ristić MM, Joseph A (2011). Marshall-Olkin bivariate Weibull distributions and processes. Statistical Papers, 52(4): 789-798.

Li X, Pellerey F (2011). Generalized Marshall-Olkin distributions and related bivariate aging properties. Journal of Multivariate Analysis, 102(10): 1399-1409.

Lin CT, Balakrishnan N (2011). Asymptotic properties of maximum likelihood estimators based on progressive Type-II censoring. Metrika, 74(3): 349-360.

Marshall AW, Olkin I (1967). A multivariate exponential distribution. Journal of the American Statistical Association, 62(317): 30-44.

Mirhosseini SM, Amini M, Kundu D, Dolati A (2015). On a new absolutely continuous bivariate generalized exponential distribution. Statistical Methods \&5 Applications, 24(1): 61-83.

Muhammed HZ (2016). Bivariate inverse Weibull distribution. Journal of Statistical Computation and Simulation, 86(12): 2335-2345.

Özel G (2015). Bivariate Kumaraswamy distribution with an application on earthquake data. In: AIP Conference Proceedings, volume 1648, 610002. AIP Publishing LLC.

Ozkut M, Bayramoglu I (2014). On marshall-Olkin type distribution with effect of shock magnitude. Journal of Computational and Applied Mathematics, 271: 150-162.

Regoli G (2009). A class of bivariate exponential distributions. Journal of Multivariate Analysis, 100(6): 1261-1269.

Sankaran P, Gleeja V (2006). On bivariate reversed hazard rates. Journal of the Japan Statistical Society, 36(2): 213-224.

Sarhan AM, Balakrishnan N (2007). A new class of bivariate distributions and its mixture. Journal of Multivariate Analysis, 98(7): 1508-1527. 